\title{
Novel starter cultures to inhibit biogenic amines accumulation during fish sauce fermentation.
}

\begin{abstract}
Bacteria with amine oxidase activity have become a particular interest to reduce biogenic amines concentration in food products such as meat and fish sausages. However, little information is available regarding the application of these bacteria in fish sauce. Hence, our study was aimed to investigate the effect of such starter cultures in reducing biogenic amines accumulation during fish sauce fermentation. Staphylococcus carnosus FS19 and Bacillus amyloliquefaciens FS05 isolated from fish sauce which possess amine oxidase activity were used as starter cultures in this study. Fermentation was held for 120 . days at $35^{\circ} \mathrm{C}$. The $\mathrm{pH}$ value increased in all samples, while salt concentration remained constant throughout fermentation. Aerobic bacteria count was significantly lower $(p<0.05)$ in the control than in inoculated samples as a result of starter cultures addition. However, it decreased during fermentation due to the growth inhibition by high salt concentration. Proteolytic bacterial count decreased during fermentation with no significant difference ( $p>0.05)$ among samples. These bacteria hydrolyzed protein in anchovy to produce free amino acid precursors for amines formation by decarboxylase bacteria. The presence of biogenic amines producing bacteria in this study was considered to be indigenous from raw material or contamination during fermentation, since our cultures were negative histamine producers. Amino acid histidine, arginine, lysine and tyrosine concentration decreased at different rates during fermentation as they were converted into their respective amines. In general, biogenic amines concentration namely histamine, putrescine, cadaverine and tyramine increased throughout fermentation. However, their concentrations were markedly higher $(\mathrm{p}<0.05)$ in the control (without starter cultures) as compared to the samples treated with starter cultures. Histamine concentration was reduced by $27.7 \%$ and $15.4 \%$ by Staphylococcus carnosus FS19 and Bacillus amyloliquefaciens FS05, respectively. Both cultures could also reduce other amines during fermentation. After 120. days of fermentation, the overall biogenic amines concentration was $15.9 \%$ and $12.5 \%$ less in samples inoculated with Staphylococcus carnosus FS19 and Bacillus amyloliquefaciens FS05, respectively, as compared to control samples. These findings emphasized that application of starter cultures with amines oxidase activity in fish sauce fermentation was found to be effective in reducing biogenic amines accumulation.
\end{abstract}

Keyword: Biogenic amines; Fish sauce; Starter culture; Biogenic amines degradation; Staphylococcus carnosus; Bacillus amyloliquefaciens. 